\title{
New simple decontamination method improves microscopic detection and culture of mycobacteria in clinical practice
}

Nora Morcillo'

Belén Imperiale'

Juan Carlos Palomino²

'Reference Laboratory of Tuberculosis

Control Program of Buenos

Aires Province, Dr. Cetrángolo

Hospital, Buenos Aires, Argentina;

${ }^{2}$ Mycobacteriology Unit Institute

of Tropical Medicine, Antwerp, Belgium
Correspondence: Nora Morcillo

Dr. Cetrángolo Hospital, Italia I750

(1602) Vicente Lopez, Buenos Aires,

Argentina

Tel +54 II $47 \mid 80939$

Fax +54 II 472 I 9153

Email nora_morcillo@yahoo.com.ar
Abstract: This study was carried out at Dr. Cetrángolo Hospital, Buenos Aires, Argentina. The objective was to compare two digestion-decontamination procedures: the N-acetyl-Lcysteine-sodium citrate- $\mathrm{NaOH}(\mathrm{NALC}-\mathrm{NaOH})$ and a combination of $7 \% \mathrm{NaCl}$ plus $\mathrm{NaOH}$, the hypertonic saline-sodium hydroxide (HS-SH) method, in detection and recovery of mycobacteria. Microscopy detection rates before and after concentration of specimens by both methods, were also compared. The study had two phases. Phase I: comparison of the gold standard NALC-NaOH and HS-SH on paired samples involving respiratory clinical specimens by means of receiver operating characteristic curve analysis. Phase II: blinded, randomized trial to assess the performance of HS-SH versus NALC-NaOH in clinical practice. Phase I: Positive microscopy rate was significantly increased in around $2.2 \%$ after concentration in comparison to that of specimens without concentration. The calculated sensitivity values for microscopy detection increased between $15.2 \%$ (HS-SH: $73.5 \%$ ) to $16.7 \%$ (NALC-NaOH: $75.0 \%$ ) over those without concentration $(58.3 \%)$. Phase II: similar diagnostic rates by microscopy and cultures were obtained by either HS-SH or NALC-NaOH. The clinical performances were also very similar. These results and the low cost of the HS-SH procedure indicate the possibility of its implementation in clinical laboratories with high burden of tuberculosis cases and low resources.

Keywords: tuberculosis, diagnosis, clinical practice

\section{Introduction}

Mycobacterium tuberculosis, the causative agent of tuberculosis (TB), infects almost the third part of the world population and kills around two million people worldwide each year. About $80 \%$ of the global TB burden occurs in low-income countries, where pulmonary disease and its transmission are most serious public health problems (Kent and Kubica 1985; IUATLD 2005). Among bacterial pathogens of man, M. tuberculosis is best known for its slow growth rate and its acid-fast lipid-rich cell wall. Culture of mycobacteria is too slow for practical diagnosis, while their acid-fastness allows rapid detection in clinical specimens (Kent and Kubica 1985; IUATLD 2000).

So far, acid-fast bacilli (AFB) detection by smear microscopy is still the most used amongst all methods currently employed worldwide in clinical laboratories for TB diagnosis on account of its simplicity, speed, low cost, and minimal requirement of equipment and technical skills. However it lacks sensitivity since a load of about 5,000 to 10,000 bacilli $/ \mathrm{mL}$ of specimen is required to give a positive result after Ziehl-Neelsen staining (WHO 1998; Liehardt and Cook 2005).

Culture on Löwenstein-Jensen solid medium requires about 10 bacilli/mL of specimen for recovery of mycobacteria, and is the gold standard for microbiological diagnosis of TB in developing countries. Nevertheless the slow growth rate of the 
pathogen leads to a delay of 4-6 weeks in obtaining a definitive diagnosis (WHO 1998; IUATLD 2000).

Several tools have been tested for rapid detection of mycobacterial growth. Automated and semi-automated culture systems based on liquid media using radioactive materials, oxygen-quenching or redox reagents have been developed. Albeit most of these systems allow results as early as 5-7 days, they are still very costly for disease-endemic and low-income countries (Roberts et al 1988; Brunello et al 1999; Palomino et al 1999). In addition they require, prior to cultivation of respiratory specimens, that they be treated by the most commonly used digestion-decontamination method, $\mathrm{N}$-acetyl-L-cysteine-sodium citrate- $\mathrm{NaOH}$ (NALC-NaOH), which also increases the total cost of the culture considerably (Kubica et al 1963; Palomino et al 1999). In this mixture, N-acetyl-L-cysteine liquefies respiratory specimens, which contain high proportions of mucus, while $\mathrm{NaOH}$ kills the contaminant bacteria present in such specimens. This method has also been recommended for inoculation of liquid media and recovery of nontuberculosis mycobacteria from different clinical specimens (Kent and Kubica 1985; Palomino et al 1999).

Since smear microscopy remains the cornerstone of TB diagnosis, operational research of sputum concentration techniques is a priority of TB control programs (IUATLD 2005). A more sensitive smear microscopy and a cheaper digestion-decontamination method would both be useful in clinic laboratories in order to achieve increased, rapid, and accurate TB diagnosis.

In this study the NALC-NaOH method was used as the gold standard and its results were compared to an alternate procedure for digestion-decontamination: the combination of hypertonic sodium chloride and sodium hydroxide (HS-SH) method as described previously (Ganoza et al 2003). In this mixture the mucolytic activity is exerted by the hypertonic $\mathrm{NaCl}$ solution.

The evaluation was designed in two phases. Phase I compared the two digestion-decontamination solutions, NALC-NaOH and HS-SH, on a paired sample basis involving respiratory clinical specimens. Phase II was a blinded and randomized trial which compared the performance of the two digestion-decontamination methods in diagnosing TB in patients.

\section{Methods}

\section{Phase I}

During a period of six month, a total of 683 respiratory clinical specimens were collected as: sputa (S), 595; bronchial washings (BW), 60; broncho-alveolar lavages (BAL), 2; and gastric aspirates (GA), 26. The rates for AFB detection by direct smear examination before and after the concentration of the specimens by each method were also compared.

In a first step, sputum smears were prepared for direct AFB examination using Ziehl-Neelsen staining, and observed at $1000 \times$ magnification. The remaining portion of each sample was divided into two equal parts and transferred to $20 \mathrm{~mL}$ plastic conical tubes. Decontamination was made simultaneously by the N-acetyl-L-cysteine and $4 \% \mathrm{NaOH}$ (NALC-NaOH) following standard procedures, and the hypertonic saline, $7.0 \% \mathrm{NaCl}$ plus $\mathrm{NaOH}$ (HS-SH). The final concentration of $\mathrm{NaOH}$ in both solutions was approximately $1.0 \%(2,8,9)$.

\section{$\mathrm{NALC}-\mathrm{NaOH}$ procedure}

Two $\mathrm{mL}$ of a mixture composed by $1.0 \mathrm{~mL} 1.0 \% \mathrm{~N}$-acetylL-cysteine (Merck, Germany) in 2.9\% citric acid (ANEDRA,

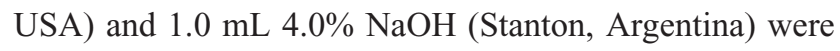
added to $2 \mathrm{~mL}$ volumes of each respiratory specimen and vortexed in a tube for $15-20$ seconds and incubated at $37^{\circ} \mathrm{C}$ for 20 minutes (Becton Dickinson 2004). Phosphate buffer pH 6.8 (Stanton) was then added and the tubes centrifuged at $3000 \mathrm{~g}$ for 15 minutes. The supernatant was then carefully discarded, and the sediment resuspended in $1-2 \mathrm{~mL}$ of phosphate buffer $\mathrm{pH}$ 6.8. This last suspension was used to inoculate culture media and to prepare smears for microscopic examination.

\section{$\mathrm{HS}-\mathrm{SH}$ procedure}

To a $2 \mathrm{~mL}$ volume of the respiratory specimen were added $2 \mathrm{~mL}$ of $7 \% \mathrm{NaCl}$ (Stanton) and $1.5 \mathrm{~mL}$ of $4 \% \mathrm{NaOH}$ and the tube mixed for 15-20 seconds and then incubated at $37^{\circ} \mathrm{C}$ for $20 \mathrm{~min}$. After this period, phosphate buffer $\mathrm{pH} 6.8$ was added and the tube centrifuged at $3000 \mathrm{~g}$ for $15 \mathrm{~min}$. The supernatant was carefully discarded and the sediment used for preparing the smears and inoculating culture media.

Smears were prepared with $200 \mu \mathrm{L}$ of each of the final decontaminated volumes and stained with the Ziehl-Neelsen stain for microscopical examination.

Also, $200 \mu \mathrm{L}$ were taken from each decontaminate and inoculated onto Löwenstein-Jensen (LJ) slants in duplicate, and $500 \mu \mathrm{L}$ were inoculated into MGIT 960 tubes (Becton Dickinson, Buenos Aires, Argentina). LJ slants were incubated at $37{ }^{\circ} \mathrm{C}$ in normal atmosphere for 60 days while MGIT960 system tubes were incubated and automatically 
read for a total period of 42 days (Palomino et al 1999; Becton Dickinson 2004).

\section{Phase II}

This phase was designed as a blinded and randomized trial (after completion of Phase I) to compare the two digestion-decontamination methods in patients suspected of TB. Respiratory specimens were blinded and randomly assigned to be treated by either NALC-NaOH or HS-SH during a one-year period.

Table 1 shows the number and percentage of Phase II clinical specimens, which were treated by NALC-NaOH or HS-SH. After arriving in the laboratory the specimens were labeled by the administrative personnel and then transferred to the technicians in charge of the smears and culture procedures. Once a week the same personnel changed the digestiondecontamination method used. Another person, to whom the method used was completely unknown, did the microscopic examination of the smears. After being obtained, samples were also transferred to $20 \mathrm{~mL}$ plastic conical tubes and treated with either NALC-NaOH or HS-SH as described. Smears and cultures were prepared and the latter incubated as mentioned above. During the experimental phase of the study, all sample volumes, speed, and duration of centrifugation were the same to allow a better comparison of the methods being tested.

\section{Statistical analysis}

The MedCalc version 9.5.2.0 (MedCalc, Mariakerke, Belgium) software was used to obtain the summary statistics in both groups of clinical isolates and to perform the comparison of paired-sample results (Altman 1992). Additionally, sensitivity, specificity, predictive values, and area under the curve were also calculated using the receiver operating characteristic (ROC) curve method (McNeil and Hanley 1984; van Der Schouw et al 1995).

Table I Number and percentage of respiratory specimens included in the Phase II of the study

\begin{tabular}{lllll}
\hline Clinical specimen & HS-SH & \multicolumn{2}{l}{ NALC-NaOH } \\
\hline & $\mathbf{N}^{\circ}$ & $\mathbf{( \% )}$ & $\mathbf{N}^{\circ}$ & $\mathbf{( \% )}$ \\
S & 1837 & 90.4 & $119 \mathrm{I}$ & 87.7 \\
BAL & 38 & 1.9 & 28 & 2.0 \\
BW & 139 & 6.9 & 122 & 9.1 \\
GA & 17 & 0.8 & 16 & 1.2 \\
Total & 2031 & 100 & 1357 & 100 \\
\hline
\end{tabular}

Abbreviations: S, sputum; BAL, bronchioalveolar lavage; BW, bronchial washing; $\mathrm{GA}$, gastric aspirate; $\mathrm{HS}-\mathrm{SH}$, hypertonic saline - sodium hydroxide, $\mathrm{NaCl}$ plus $\mathrm{NaOH}$; NALC, N-acetyl-L-cysteine - sodium citrate plus $\mathrm{NaOH}$.

\section{Results and discussion}

Phase I

A total of 96 (14.1\%) clinical specimens were detected as positive by smear examination and/or culture. Table 2 shows the distribution of positive, negative, and contaminated cultures and AFB direct smear examination results from the specimens included in this phase of the study. Similar results for both AFB smear examination and cultures were obtained by the two concentration methods.

The positive rates for AFB detection by HS-SH and NALC-NaOH were $10.4 \%$ (71/683) and 10.5\% (72/683), respectively $\left(\chi^{2}=0.064 ; \mathrm{P}=0.7996\right)$, while this rate for $\mathrm{AFB}$ smear examination before the concentration of the specimens was $8.2 \%(56 / 683)$ (HS-SH difference, $\chi^{2}=0.013 ; \mathrm{P}=0.9081$; NALC-NaOH difference, $\chi^{2}=0.019 ; \mathrm{P}=0.8915$ ).

Table 3 shows the statistical parameters found from the analysis of the results obtained by these two methods with paired samples. Sensitivity (S) for HS-SH and NALC$\mathrm{NaOH}$ was $73.5 \%$ and $75.0 \%$ for microscopy examination $\left(\chi^{2}=0.330, \mathrm{P}=0.5673\right)$ while specificity (SP) was $99.5 \%$ and $99.3 \%$, respectively $\left(\chi^{2}=0.016, \mathrm{P}=0.8980\right)$. When $\mathrm{S}$ was calculated from direct AFB smear examination before specimen concentration, a value of $58.3 \%$ was obtained. The differences between this value and those obtained by both HS-SH $\left(\chi^{2}=4.282 ; \mathrm{P}=0.0385\right)$ and NALC-NaOH $\left(\chi^{2}=5.295, \mathrm{P}=0.0214\right)$ were significant.

The analysis of the culture results showed that similar values of sensitivity and specificity were found when using LJ (HS-SH, S: $88.4 \%$ and SP: 100.0\%; NALC-NaOH, S: $88.3 \%$ and SP: $99.8 \%$ ) and MGIT960 (HS-SH, S: $92.6 \%$, SP: $100.0 \%$; NALC-NaOH, S: $91.5 \%$ and SP: $99.8 \%$ ), although the global contamination rate for specimens treated by NALC-NaOH (MGIT 2.1\%, LJ, 2.5\%) was higher than those by HS-SH (MGIT 1.2\%, LJ: 1.2\%). No significant difference was observed in S, SP, area under the ROC curve and predictive values between NALC-NaOH and HS-SH results. The TB incidence rate (49 per 100,000 inhabitants, based on the number of notified cases) as an estimation of the north Buenos Aires region prevalence during the study period was used for predictive value calculations.

\section{Phase II}

Results are summarized in Table 4. Using HS-SH and NALC-NaOH solutions it was possible to diagnose $323(15.9 \%)$ and $219(16.1 \%)$ TB cases respectively $\left(\chi^{2}=0.003, \mathrm{P}=0.9545\right)$. From these figures, 64.8\% (142/219) and $65.9 \%(213 / 323)$ of the cases were diagnosed by AFB with NALC-NaOH and $\mathrm{HS}-\mathrm{SH}$ respectively 
Table 2 Phase I: Distribution of direct smear examination and culture results using paired samples from 683 clinical specimens

\begin{tabular}{|c|c|c|c|c|c|c|}
\hline \multirow[b]{3}{*}{ Result } & \multicolumn{6}{|c|}{ Technique and decontamination procedure } \\
\hline & \multicolumn{2}{|l|}{ AFB } & \multicolumn{2}{|l|}{ MGIT960 } & \multicolumn{2}{|l|}{ LJ } \\
\hline & $\begin{array}{l}\text { HS-SH } \\
\left.\text { No }^{\circ} \%\right)\end{array}$ & $\begin{array}{l}\text { NALC-NaOH } \\
\mathbf{N}^{\circ}(\%)\end{array}$ & $\begin{array}{l}\text { HS-SH } \\
\mathbf{N}^{\circ}(\%)\end{array}$ & $\begin{array}{l}\text { NALC-NaOH } \\
N^{\circ}(\%)\end{array}$ & $\begin{array}{l}\text { HS-SH } \\
\left.\text { No }^{\circ} \%\right)\end{array}$ & $\begin{array}{l}\text { NALC-NaOH } \\
\mathbf{N}^{\circ}(\%)\end{array}$ \\
\hline Positive & $71(10.4)$ & $72(10.5)$ & $88(12.9)$ & $87(12.7)$ & $84(12.3)$ & $84(12.3)$ \\
\hline Negative & $612(89.6)$ & $611(89.5)$ & $587(85.9)$ & $582(85.2)$ & $591(86.5)$ & $582(85.2)$ \\
\hline Contaminated & - & - & $8(1.2)$ & $14(2.1)$ & $8(1.2)$ & $17(2.5)$ \\
\hline
\end{tabular}

Abbreviations: AFB, smear examination; $\mathrm{HS}$-SH, hypertonic saline - sodium hydroxide, $\mathrm{NaCl}$ plus $\mathrm{NaOH} ; \mathrm{NALC}, \mathrm{N}$-acetyl-L-cysteine - sodium citrate plus $\mathrm{NaOH}$; $\mathrm{MGIT960}$, culture system; LJ, Löwenstein-Jensen solid egg-based medium.

$\left(\chi^{2}=0.030, \mathrm{P}=0.8633\right)$. The overall yield of the cultures from specimens treated by NALC-NaOH and HS-SH were $35.2 \%(77 / 219)$ and $34.0 \%(110 / 323)$ respectively $\left(\chi^{2}=0.039, \mathrm{P}=0.8443\right)$.

Table 4 shows that the only significant difference between results of the two methods was the proportion of contaminated cultures from AFB-positive specimens, $0.3 \%$ for HS-SH and $1.0 \%$ for NALC-NaOH, $\left(\chi^{2}=7.000\right.$, $\mathrm{P}=0.0082$ ).

The HS-SH method showed higher sensitivity for microscopic detection of mycobacteria in comparison with NALC$\mathrm{NaOH}$, but the difference was not significant $(7.6 \%$ vs $6.2 \%$, $\mathrm{P}=0.8904)$. Specificity, accuracy and predictive values were obtained by analyzing culture results processed by NALC-NaOH and HS-SH. These values also showed a high correlation with each other (see Table 5).

Rapid diagnosis of TB is becoming increasingly important to improve cure rates, lower the risk of airborne disease spread, and to prevent the transmission of emergent drugresistant strains of $M$. tuberculosis and its severe implications, which are worse in HIV-infected patients.

During the last decade several methods, based on a wide spectrum of decontamination and digestion reagents, with differences in the time they consume and in their cost have been reported. These methods for preparing samples for smear microscopy and cultivation of $M$. tuberculosis involve the use of $4 \% \mathrm{NaOH} ; 0.5 \%$ NALC and $2 \% \mathrm{NaOH}$; dithiothreitol and $2 \% \mathrm{NaOH} ; 13 \%$ trisodium phosphate with or without benzalkonium chloride (Zephiran); 5\% oxalic acid; $1 \%$ cetylpyridium chloride and $2 \% \mathrm{NaCl}$ (Smithwick et al 1975; Roberts et al 1988; Whittier et al 1993; Rattan et al 1994). A method for processing respiratory specimens by using Cis-Carboxypropylbetaine (CB-18w), a zwitterionic detergent has also been described for detection of mycobacteria (Thornton et al 1998). Household bleach (NaOCI) has also been used alone, for liquefaction of sputum and microscopy (van Deun et al 2000; Ramsay et al 2006). It uses overnight sedimentation, thus eliminating the use of a centrifuge, and smearing from the sediment. In 3 studies performed in Ethiopia and India, the use of the NaOCI method increased the number of samples positive for acid-fast bacilli in more than $100 \%$ (Gebre et al 1995). Another method adding chitin solution for aiding mucus digestion has been described by Farnia and colleagues (2002). Sensitivity and specificity for this method were reported as $80 \%$ and $96.7 \%$ (Farnia et al 2002).

Table 3 Phase I: Statistical parameters (in percentage) sensitivity, specificity, accuracy, and predictive values calculated for both decontamination methods

\begin{tabular}{|c|c|c|c|c|c|c|c|c|c|c|}
\hline \multirow[t]{3}{*}{ Technique } & \multicolumn{10}{|c|}{ Digestion - decontaminationprocedure } \\
\hline & \multicolumn{5}{|c|}{$\begin{array}{l}\text { HS-SH } \\
\text { (Values in \%) }\end{array}$} & \multicolumn{5}{|c|}{$\begin{array}{l}\text { NALC-NaOH } \\
\text { (Values in \%) }\end{array}$} \\
\hline & $\mathbf{S}$ & $\mathbf{S P}$ & AUC & PPV & NPV & $\mathbf{S}$ & SP & AUC & PPV & NPV \\
\hline AFB & 73.5 & 99.5 & 81.0 & 95.2 & 94.1 & 75.0 & 99.3 & 81.0 & 93.5 & 94.2 \\
\hline LJ & 88.4 & 100 & 94.0 & 100 & 98.1 & 88.3 & 99.8 & 94.0 & 98.6 & 99.1 \\
\hline MGIT & 92.6 & 100 & 96.0 & 100 & 98.8 & 91.5 & 99.8 & 96.0 & 98.7 & 98.6 \\
\hline
\end{tabular}

Abbreviations: AFB, smear examination; MGIT960, culture system; LJ, Löwenstein-Jensen solid medium; $\mathrm{HS}-\mathrm{SH}$, hypertonic saline - sodium hydroxide, $\mathrm{NaCl}$ plus $\mathrm{NaOH}$; NALC, N-acetyl-L-cysteine - sodium citrate plus $\mathrm{NaOH}$; S, sensitivity; SP, specificity; PPV and NPV, positive and negative predictive values; AUC, area under receiver operating characteristic curve. 
Table 4 Distribution of smears and culture results obtained from the specimens included in Phase II of the study and treated by HS-SH and NALC-NaOH, respectively

\begin{tabular}{|c|c|c|c|c|c|c|}
\hline \multirow{4}{*}{$\begin{array}{l}\text { Smear } \\
\text { and culture } \\
\text { results }\end{array}$} & \multicolumn{4}{|c|}{ Specimens } & \multicolumn{2}{|c|}{ Statistics } \\
\hline & \multicolumn{6}{|c|}{ Decontamination/liquefaction Method } \\
\hline & \multicolumn{2}{|c|}{ HS-SH } & \multicolumn{2}{|c|}{ NALC-NaOH } & \multirow[t]{2}{*}{$\chi^{2}$} & \multirow[t]{2}{*}{$P$ value } \\
\hline & $\overline{\mathbf{N}^{\circ}}$ & $\%$ & $\mathbf{N}^{\circ}$ & $\%$ & & \\
\hline AFB $+/ C-$ & 30 & 1.5 & 14 & 1.0 & 1.620 & 0.2031 \\
\hline $\mathrm{AFB}+/ \mathrm{C}+$ & 177 & 8.7 & 115 & 8.5 & 0.024 & 0.8777 \\
\hline AFB-/C+ & 110 & 5.4 & 77 & 5.6 & 0.071 & 0.7893 \\
\hline AFB-/Cc & 89 & 4.4 & 75 & 5.5 & 0.001 & 0.9697 \\
\hline $\mathrm{AFB}+/ \mathrm{Cc}$ & 6 & 0.3 & 13 & 1.0 & 7.000 & 0.0082 \\
\hline AFB-/C- & 1619 & 79.8 & 1063 & 78.3 & 0.789 & 0.3743 \\
\hline Total & 2031 & 100.0 & 1357 & 100.0 & - & - \\
\hline
\end{tabular}

Abbreviations: AFB, acid-fast bacilli direct examination; C, culture; + and -, positive and negative results; c, contaminated; HS-SH, hypertonic saline-sodium hydroxide, 7\% $\mathrm{NaCl}$ plus $\mathrm{NaOH}$; NALC-NaOH, $\mathrm{N}$-acetyl-L-cysteine - sodium citrate plus $\mathrm{NaOH}$.

Several decontamination procedures require that the performance conditions - such as the exposure time to trisodium phosphate - be carefully controlled. Zephiran requires neutralization with lecithin and is not compatible with inoculation on egg-based culture media; the usefulness of chitin solutions for culture has not been evaluated yet; bleach solution is of course unsuitable for cultivation. For the recovery of $M$. tuberculosis after treatment with CB-18w, a separate protocol with three lytic enzymes, which increases the whole cost, is used in conjunction with CB-18w. However, the initial studies showed an important increase in sensitivity with this method when compared to the NALC-NaOH method (Thornton et al 1998; Scott et al 2002).

Another recently published method describes the use of phenol and ammonium sulphate combination (PhAS) for liquefaction of sputum. Besides the fact that this method needs an overnight sedimentation, it kills the mycobacteria, so it is not suitable for culture. Sensitivity and specificity of these methods were reported as high as $85 \%$ and $97 \%$, respectively (Selvakumar et al 2002).

In this study the AFB positive rate was significantly increased by about $2.2 \%$ after the concentration procedures in comparison to those without such treatment $(10.4 \%$ and $10.5 \%$ vs $8.2 \%$ ). Furthermore and independently of the method used, the calculated S values for AFB detection increased between $15.2 \%$ (HS-SH-S: $73.5 \%$ ) to $16.7 \%$ (NALC-NaOH-S: 75.0\%) above the value obtained without specimen concentration $(58.3 \%)$.

The analysis of culture results showed that similar values were obtained when using LJ and MGIT960 after applying either HS-SH or NALC-NaOh methods.

The clinical performance of the HS-SH solution was assessed in the Phase II study by ROC curve analysis of the results. The overall technical performances were also very similar.

In our country the cost of the NALC-NaOH method is about US\$1.0 per clinical specimen when homemade

Table 5 Sensitivity, specificity, accuracy, predictive values, and positive rates calculated for both decontamination methods

\begin{tabular}{|c|c|c|c|c|c|c|}
\hline \multirow[t]{2}{*}{ sv } & \multicolumn{2}{|c|}{ Technique for AFB } & \multirow{2}{*}{$\frac{\text { Difference }}{\chi^{2}(\mathbf{P})}$} & \multicolumn{2}{|c|}{ Technique for Culture } & \multirow{2}{*}{$\frac{\text { Difference }}{\chi^{2}(\mathbf{P})}$} \\
\hline & HS-SH & NALC-NaOH & & HS-SH & NALC-NaOH & \\
\hline$S$ & 73.5 & 75.0 & $0.877(0.3490)$ & 92.6 & 91.5 & $1.214(0.2705)$ \\
\hline SP & 99.5 & 99.3 & 0.271 (0.6029) & 100.0 & 99.8 & $1.951(0.1625)$ \\
\hline AUC & 81.0 & 81.0 & $0.002(0.9644)$ & 96.0 & 96.0 & $0.008(0.9287)$ \\
\hline PPV & 95.2 & 93.5 & $4.216(0.0400)$ & 100.0 & 98.7 & $24.091(<0.0001)$ \\
\hline NPV & 94.1 & 94.2 & $0.002(0.9627)$ & 98.8 & 98.6 & $0.124(0.7252)$ \\
\hline
\end{tabular}

Abbreviations: SV, statistical value; S, sensitivity; SP, specificity; PPV and NPV, positive and negative predictive value; $\mathrm{HS}-\mathrm{SH}$, hypertonic saline sodium hydroxide, $7 \%$ NaCl plus $\mathrm{NaOH}$; NALC-NaOH, N-acetyl-L-cysteine - sodium citrate plus $\mathrm{NaOH}$. 
and US\$3.5 when using commercial kits (MycoPrep; Becton Dickinson, MD, USA). The cost when using HS-SH solutions was about US\$0.25 per sample. Besides, the HS-SH method is easy to set up in clinical laboratories devoted to detection and recovery of mycobacteria on LJ slants, since there is no need for special training of technical personnel.

The results obtained as well as the extremely low cost of the HS-SH digestion-decontamination procedure make its implementation possible in clinical laboratories with high burdens of TB cases and low resources.

\section{Acknowledgments}

We would like to thank Dr. Humberto Guerra (Universidad Peruana Cayetano Heredia) for his kind advice during the preparation of the manuscript, and Mr. Daniel Cardinali, Mr. Marcelo Mazza, Mr. Juan Carlos Donato, and Mrs. Zuni Zubov for their technical support. This study was partially funded by the European Commission projects: ICA4-CT-2001-10087 and LSHP-CT-2004-516028.

\section{References}

Altman D. 1992. Basic methods for comparison of groups. Practical statistical for medical research. 2nd ed. London: Chapman and Hall, pp. 274-94.

Becton Dickinson Co. 2004. Insert: BBLTM MGIT Mycobacteria Growth Indicator Tube. Insert kit, 1999. CE L000180JAA(E)2004/06. Becton Dickinson Co., USA.

Brunello F, Favari F, Fontana R. 1999. Comparison of MB/Bact T and BACTEC 460 TB systems for recovery of mycobacteria from various clinical specimens. J Clin Microbiol, 26:1206-9.

Farnia P, Mohammadi F, Zarifi Z, et al. 2002. Improving sensitivity of direct microscopy for detection of acid-fast bacilli in sputum: use of chitin in mucus digestion. J Clin Microbiol, 40:508-11.

Ganoza C, Ricaldi J, Agapito J, et al. 2003. [Decontaminación y concentración con salino hipertónico-hidróxido de sodio (SH-HS) para microscopía y cultivo de Mycobacterium tuberculosis en muestras de esputo.] Universidad Peruana C. Heredia and Hospital Nacional Cayetano Heredia XIV Jornadas Científicas -Raúl León Barúa [online]. Accessed on March 11, 2008. URL: http://www.upch.edu.pe/duiict/ jornadas/jornaxiv/jornaxiv.asp.

Gebre N, Karlsson U, Jonsson G, et al. 1995. Improved microscopical diagnosis of pulmonary tuberculosis in developing countries. Trans R Soc Trop Med Hyg, 89:191-3.

[IUATLD] International Union Against Tuberculosis and Lung Disease. 2000. Sputum examination for tuberculosis by direct microscopy in low income countries. 5th Edition. Technical Guide, Paris: International Union Against Tuberculosis and Lung Disease.

[IUATLD] Tuberculosis Division, International Union Against Tuberculosis and Lung Disease. 2005. Tuberculosis bacteriology-priorities and indications in high prevalence countries: position of the technical staff of the Tuberculosis. Division of the International Union against Tuberculosis and Lung Disease. Int J Tuberc Lung Dis, 9:355-61.
Kent PT, Kubica GPW. 1985. Public Health Mycobacteriology. A guide for the level III Laboratory. Atlanta: Centers for Disease Control.

Kubica GPW, Dye E, Cohn ML, et al. 1963. Sputum digestion and decontamination with N-acetyl-L cysteine sodium hydroxide for culture of mycobacteria. Am Rev Respir Dis, 87:775-9.

Liehardt C, Cook SV. 2005. Conducting clinical trials on tuberculosis in resource-poor countries: imposed science or equally shared efforts? Int J Tuberc Lung Dis, 9:943.

McNeil BJ, Hanley JA. 1984. Statistical approaches to the analysis of receiver operating characteristic (ROC) curves. Med Decis Making, 4:137-50.

Palomino JC, Traore H, Fissette K, et al. 1999. Evaluation of mycobacterial growth indicator tube (MGIT) for drug susceptibility testing of Mycobacterium tuberculosis. Int J Tuberc Lung Dis, 3:344-8.

Ramsay A, Squire SB, Siddiqi K, et al. 2006. The bleach microscopy method and case detection for tuberculosis control. Int J Tuberc Lung Dis, 10:256-8.

Rattan A, Kishore K, Singh S, et al. 1994. Evaluation of a safe sputum processing method for detecting tuberculosis. J Clin Pathol, 47:411-3.

Roberts GD, Goodman NL, Heifets LB, et al. 1988. Evaluation of the BACTEC radiometric method for recovery of mycobacteria and drug susceptibility testing of Mycobacterium tuberculosis from acid-fast smear-positive specimens. J Clin Microbiol, 15:689-96.

Roberts GD, Koneman EW, Kim YK. 1991. Mycobacterium. In: Balows A, Hauster Jr. WJ, Herrmann KL, et al. (eds). Manual of Clinical Microbiology, 1st ed. Washington, DC: American Society for Microbiology. pp. 304-9.

Scott CP, dos Anjos Filho L, Carvalho de Queiroz Mello F, et al. 2002. Comparison of C18-carboxypropylbetaine and standard N-AcetylL-Cysteine- $\mathrm{NaOH}$ processing of respiratory specimens for increasing tuberculosis smear sensitivity in Brazil. J Clin Microbiol, 40:3219-22.

Selvakumar N, Rahman F, Garg R, et al. 2002. Evaluation of the phenol ammonium sulfate sedimentation smear microscopy method for diagnosis of pulmonary tuberculosis. J Clin Microbiol, 40:3017-20.

Smithwick RW, Stratigos CB, David HL. 1975. Use of cetylpyridinium chloride and sodium chloride for the decontamination of sputum specimens that are transported to the laboratory for the isolation of Mycobacterium tuberculosis. J Clin Microbiol, 1:411-3.

Thornton CG, MacLellan KM, Brink TL, et al. 1998a. Novel method for processing respiratory specimens for detection of mycobacteria by using C18-Carboxypropylbetaine: blinded study. J Clin Microbiol, 36:1996-2003.

Thornton CG, MacLellan KM, Brink TL, et al. 1998b. Processing respiratory specimens with $\mathrm{C} 18$-Carboxypropylbetaine: development of a sediment resuspension buffer that contains enzymes to reduce the contamination rate and lecithin to alleviate toxicity. J Clin Microbiol, 36:2004-13.

Van Der Schouw IT, Verbeek AIM, Ruijs SHJ. 1995. Guidelines for the assessment of new diagnostic tests. Invest Radiol, 30: 334-40.

Van Deun A, Maug AK, Cooreman E, et al. 2000. Bleach sedimentation method for increased sensitivity of sputum smear microscopy: does it work? Int J Tuberc Lung Dis, 4:371-6.

Whittier S, Hopfer RL, Knowles MR, et al. 1993. Improved recovery of mycobacteria from respiratory secretions of patients with cystic fibrosis. J Clin Microbiol, 31:861-4.

[WHO] World Health Organization. 1998. Laboratory services in tuberculosis control Part II: Microscopy. WHO/TB/98.258. Geneva: World Health Organization. 